\section{Carotid stiffening predicts cardiovascular risk stratification in mid-life: non-invasive quantification with ultrafast ultrasound imaging}

\author{
Zhengqiu Zhu', Lingshan Chen ${ }^{2}$, Wenjun Liư ${ }^{3}$, Yiyun Wu', Chong Zou ${ }^{4,5}$, Xinyi Zhang ${ }^{1}$, \\ Shanshan He', Yinping Wang' ', Bixiao Shen', Xuehui Ma', Hui Gao' , Yun Luan', Hui Huang' \\ * Author affiliations appear at the end of this article.
}

Purpose: The present study investigated the association between Systematic COronary Risk Evaluation (SCORE)-estimated cardiovascular risk and carotid stiffening in a middle-aged population using ultrafast pulse wave velocity (ufPWV).

Methods: This study enrolled 683 participants without known cardiovascular disease or diabetes mellitus who underwent ufPWV measurements. Clinical interviews, physical examinations, laboratory findings, carotid intima-media thickness (CIMT), pulse wave velocity (PWV) at the beginning of systole (PWV-BS), and PWV at the end of systole (PWV-ES) were assessed. Each participant underwent an assessment of SCORE risk based on major cardiovascular risk factors (CVRFs), including age, sex, smoking, systolic blood pressure (SBP), and total cholesterol (TC). Crude and adjusted odds ratios (ORs) with 95\% confidence intervals and ordinal logistic regression were used. Overall CVRFs were adjusted to assess ORs.

Results: CIMT and carotid stiffening in PWV-BS and PWV-ES were significantly different between sex subgroups (all $P<0.05$ ), but only PWV-ES increased gradually in age and SCORE-estimated risk subgroups (all $P<0.05)$. Compared with cIMT $(r=0.388, P<0.001)$ and PWV-BS $(r=0.159$, $P<0.001)$, PWV-ES was more strongly correlated with SCORE categories $(r=0.405, P<0.001)$. Higher PWV-ES values were associated with SCORE categories independently of Sex, SBP, TC, and smoking in moderate-risk and high-risk subgroups ( $O R, 1.63 ; P<0.001$ and $O R, 2.12 ; P=0.024$, respectively), but were not independent of age in all risk subgroups (all $P>0.05$ ).

Conclusion: Carotid stiffening quantified by ufPWV is linked to SCORE categories, and elevated PWV-ES may aid in cardiovascular risk stratification.

Keywords: Atherosclerosis; Cardiovascular risk; Ultrafast ultrasound imaging; Pulse wave velocity; Arterial stiffness

Key points: Carotid stiffening quantified by ultrafast pulse wave velocity is associated with Systematic COronary Risk Evaluation (SCORE)-estimated risk. Elevated pulse wave velocity at the end of systole (PWV-ES) values may be a quantitative predictor of cardiovascular risk. The predictive role of PWV-ES is independent of some major cardiovascular risk factors, except for age.

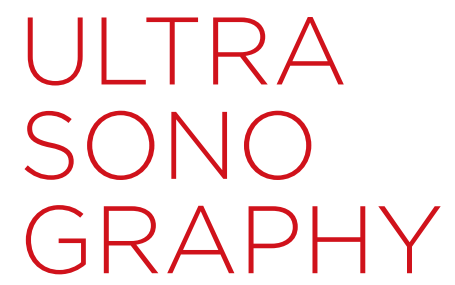

ORIGINAL ARTICLE

https://doi.org/10.14366/usg.21197 pISSN: 2288-5919 • elSSN: 2288-5943 Ultrasonography 2022;41:462-472

Received: September 15, 2021 Revised: October 25, 2021 Accepted: November 1, 2021

Correspondence to: Hui Huang, MD, Department of Ultrasound, Affiliated Hospital of Nanjing University of Chinese Medicine, Jiangsu Province Hospital of Chinese Medicine, 155 Hanzhong Road, Nanjing 210029, China

Tel. +86-25-8661-7141

Fax. +86-25-8661-7141

E-mail: szcrhh007@vip.163.com

This is an Open Access article distributed under the terms of the Creative Commons Attribution NonCommercial License (http://creativecommons.org/ licenses/by-nc/4.0/) which permits unrestricted noncommercial use, distribution, and reproduction in any medium, provided the original work is properly cited.

Copyright (C) 2022 Korean Society of Ultrasound in Medicine (KSUM)

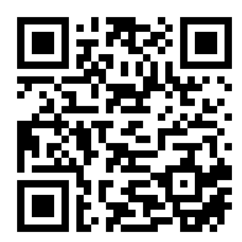

How to cite this article:

Zhu Z, Chen L, Liu W, Wu Y, Zou C, Zhang $X$, et al. Carotid stiffening predicts cardiovascular risk stratification in mid-life: non-invasive quantification with ultrafast ultrasound imaging. Ultrasonography. 2022 Jul;41(3):462-472. 


\section{Introduction}

Cardiovascular risk in asymptomatic individuals is assessed on the basis of conventional cardiovascular risk factors (CVRFs), and most cardiovascular events are linked to elevated CVRFs [1]. However, previous studies, such as the Progression of Early Subclinical Atherosclerosis (PESA) study [2], have prospectively shown that individuals with a low CVRF burden may still have subclinical atherosclerosis, especially young people and women. CVRFs underlie most cardiovascular events, and even in healthy individuals, their rational evaluation determines strategies to arrest disease development [2,3]. European guidelines recommend using Systematic COronary Risk Evaluation (SCORE) charts to assess overall 10-year cardiovascular risk for risk factor management [4]. The SCORE algorithm includes age, sex, smoking status, systolic blood pressure (SBP), and serum total cholesterol (TC), enabling a rapid calculation of risk [4]. Previous studies have shown that SCORE-estimated risk is highly associated with cardiovascular disease progression [5] and prevalent subclinical atherosclerosis [6] in the general population. However, up-to-date evidence showing that the sole use of SCORE-estimated risk stratification translates into a reduction in cardiovascular disease morbidity and mortality is scarce [7].

Increasingly many studies are reinforcing the potential value of risk assessment to detect atherogenesis using non-invasive imaging techniques, including various ultrasound modalities [8]. For decades, carotid intima-media thickness (cIMT) has been more widely explored in terms of its relationship with cardiovascular risk than direct evaluation of atheromas [9]. However, a recent large-scale meta-analysis (119 clinical trials involving 100,667 patients) revealed the final missing link supporting the usefulness of cIMT progression as a surrogate marker for cardiovascular risk [10]. Moreover, the prevalence of abnormal cIMT was low (9\%) in the PESA study, despite the overall high prevalence of early atherogenesis (63\%) [2]. Consequently, an appropriate enhanced modality is clearly needed to evaluate the subclinical phase before morphological alterations that manifest as CIMT thickening or plaques.

Atherogenesis with microstructural remodeling of arterial walls in the very early stages can lead to increased arterial stiffness $[11,12]$, which is directly associated with increased cardiovascular and all-cause mortality risk $[13,14]$. Ultrafast pulse wave velocity (ufPWV) is a novel modality of ultrafast ultrasound imaging that can assess arterial stiffening in vivo. It is non-invasive, reproducible, and relatively inexpensive [15]. Unlike all prior pulse wave velocity (PWV) techniques, such as brachial-ankle PWV or carotid-femoral PWV, ufPWV directly photographs the propagation of heart pulse waves with ultrafast speeds ( $>2,000$ frames/s). Afterwards, it calculates carotid PWV almost in real time [16]. Prior studies have shown that increased arterial stiffness detected by ufPWV is highly associated with atherosclerotic risk factors, including dyslipidemia [17], hypertension [18], diabetes mellitus [19], and coronary artery disease [20]. The authors' previous studies have shown that ufPWV can be utilized in healthy individuals with non-thickened CIMT [17] or with no major CVRFs [21] to identify notable carotid stiffening, suggesting that ufPWV could be used for the early phase of atherogenesis risk assessment. However, to date, it is still not clear whether carotid stiffness quantified by ufPWV is associated with the SCORE, which is routinely used to identify 10-year overall cardiovascular risk in clinical practice.

The aim of this study was to utilize ufPWV to quantify arterial stiffness and compare ufPWV findings with the SCORE algorithm to explore the association between arterial stiffening and the presence of overall atherogenesis risk in a middle-aged population.

\section{Materials and Methods}

\section{Compliance with Ethical Standards}

This study was approved by the ethics committee of the Affiliated Hospital of Nanjing University of Chinese Medicine (2017NL-04802 ), and the methods were carried out in accordance with the Declaration of Helsinki. Written informed consent was obtained from all patients.

\section{Study Participants}

SCORE charts are recommended for assessing apparently healthy people, not those with cardiovascular disease or at a very high or high risk of other conditions, such as diabetes mellitus [4]. In this cohort, the definition of apparently healthy people was (1) no history of known cardiovascular disease, cancer, chronic liver, autoimmune or kidney disease, or any disease expected to decrease life expectancy; and (2) an ability to undergo an ultrasonography examination of both sides of the carotid. Between May 2017 and April 2021, 1,380 apparently healthy participants were prospectively and consecutively enrolled in this study to undergo heath checkups and ufPWV examinations. Of those, 1,103 participants with available data were included in the analysis after excluding those with known cardiovascular disease or diabetes mellitus $(n=129)$ due to missing laboratory or imaging data $(n=65)$ or invalid ufPWV measurements ( $n=83$, detailed in the ufPWV protocol) (Fig. 1). To assess the association between ufPWV and SCORE, which is recommended for use in middle-aged individuals (40-65 years of age) [4], 420 individuals were excluded because they were aged $<40$ years or $>65$ years (Fig. 1). Finally, 683 participants $(49.5 \%$ 


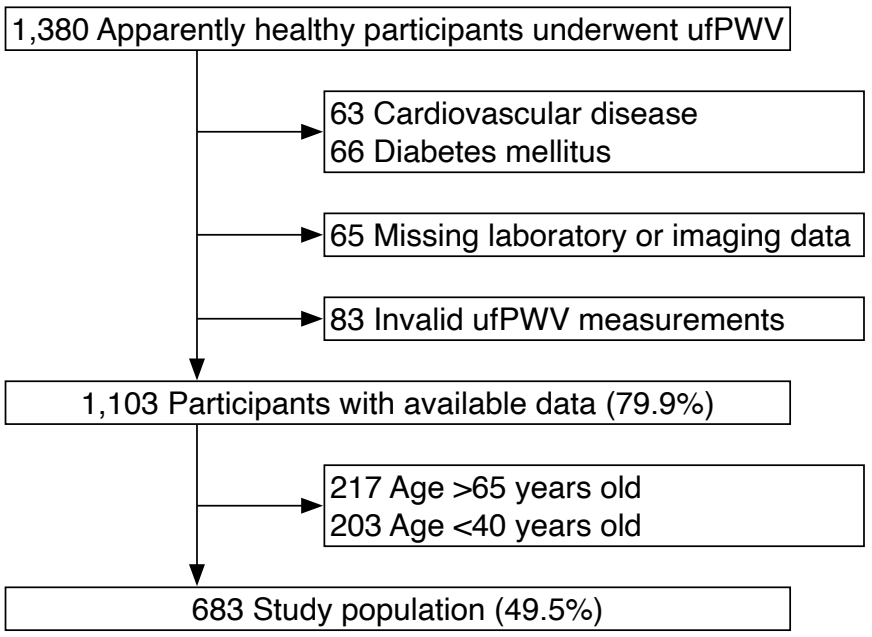

Fig. 1. Flow diagram of study cohort selection. ufPWV, ultrafast pulse wave velocity.

of all enrolled participants) were included for further analysis (Fig. 1). The estimated glomerular filtration rate (eGFR) was calculated according to the Chronic Kidney Disease Epidemiology Collaboration equation [22].

\section{SCORE}

The 2016 SCORE risk chart [4], which requires data on sex, age, smoking, SBP, and TC, was used to calculate a score for each participant. Smoking was defined in accordance with the European Society of Cardiology guidelines for cardiovascular disease prevention [4]. SBP and diastolic blood pressure (DBP) were assessed in each arm thrice using an automatic device (Omron, Kyoto, Japan). The mean highest blood pressure value from each arm was used for analysis. Laboratory findings, including hemoglobin, fasting blood glucose, uric acid, low-density lipoprotein (LDL), highdensity lipoprotein (HDL), triglyceride (TG), and TC, were analyzed in the hospital laboratory using a venous blood sample collected after an overnight (8 hours) fast. Based on the calculated SCORE, each participant was assigned to one of four risk groups: low risk, $<1 \%$; moderate risk, $1 \%-4 \%$; high risk, $5 \%-9 \%$; and very high risk, $\geq 10 \%[4]$.

\section{cIMT and ufPWV Protocol}

All carotid ultrasound examinations were performed according to a standardized protocol [23] by sonographers specifically trained in carotid ultrasound techniques. CIMT and ufPWV were performed in all participants using a Supersonic Imagine ultrasound device equipped with an SL10-2 linear array probe (Supersonic Imagine, Aix-en-Provence, France). cIMT was measured with the Aixplorer CIMT automatic measurement system (ACAMS), as described previously $[17,21]$ (Fig. 2A). The width of the automatically traced
CIMT region divided by the region of interest (ROI; $1.0 \mathrm{~cm}$ ) formed a ratio termed "fit" (Fig. 2A). A fit of $\geq 70 \%$ was considered a valid measurement. Three valid cIMT values were obtained in the left and right common carotid arteries (CCAs), respectively. For each participant, the mean of all cIMT values was recorded as the final cIMT value.

The ufPWV protocol is illustrated in Fig. 2B. The detailed ufPWV acquisition process and methodology have previously been reported by the authors of the present study, together with satisfactory validation of intra- and inter-operator reproducibility of this novel technique $[17,21]$. In brief, the probe was placed at the carotid nonplaque region to include the main CCA segment, $\sim 1.0-1.5 \mathrm{~cm}$ away from the bulb. Participants were instructed to hold their breath for 5 seconds while ufPWV was measured. After stable ufPWV imaging was achieved ( $\sim 3$ seconds), the ROI box automatically covered and tracked the carotid walls. Then, the PWV at the beginning of systole (PWV-BS), the PWV at the end of systole (PWV-ES), and the variance of each $(\Delta \pm)$ were calculated, respectively (Fig. 2B). A variance of $\leq 1.0 \mathrm{~m} / \mathrm{s}$ in ufPWV was considered indicative of a valid measurement. Invalid measurements included those with (1) failure to calculate PWV-BS or PWV-ES; (2) $\Delta \pm$ of $>1.0 \mathrm{~m} / \mathrm{s}$; (3) improper $\mathrm{ROI}$ localization with the tracing line outside of the arterial wall $[17,21]$. For each participant, three valid ufPWV measurements were recorded, and the mean of the left and right CCAs was used as the final ufPWV value.

\section{Statistical Analysis}

All statistical analyses were conducted using SPSS version 18 (SPSS Inc., Chicago, IL, USA). Baseline characteristics are presented as mean \pm standard deviation for continuous variables, and as counts and proportions for categorical variables. The distribution of continuous variables was assessed by the Kolmogorov-Smirnov Z test. Differences between normally distributed continuous variables, non-normally distributed continuous variables, and categorical variables were analyzed using the independent-sample Student t-test, Mann-Whitney $U$ test and chi-square test, respectively. The Levene homogeneity test of variance was performed, and if its P-value was $<0.05$, a corrected P-value of the t-test was reported. Histograms were performed to compare CIMT, PWV-BS, and PWVES between age and SCORE subgroups. For detailed analyses of the associations between CIMT, ufPWV, major CVRFs, and SCORE, Pearson correlation coefficients were employed (except for sex and smoking, since dichotomous variables cannot be assessed with a linear correlation analysis). Percent stacked area charts were used to assess the distribution of SCORE in age and sex subgroups. To compare the effects of different ufPWV values on SCORE-estimated risk in this cohort, univariable and multivariable analyses were 


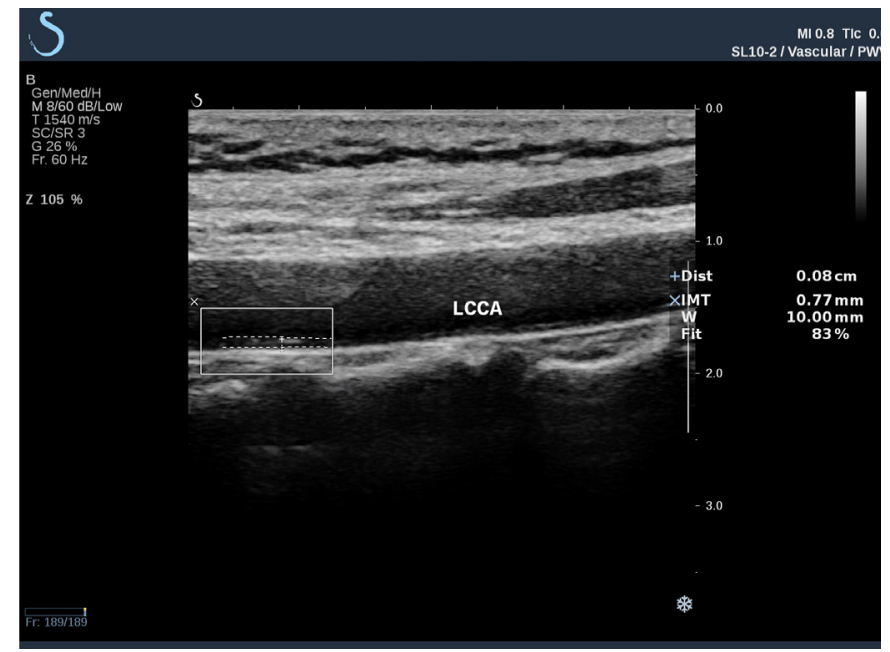

A

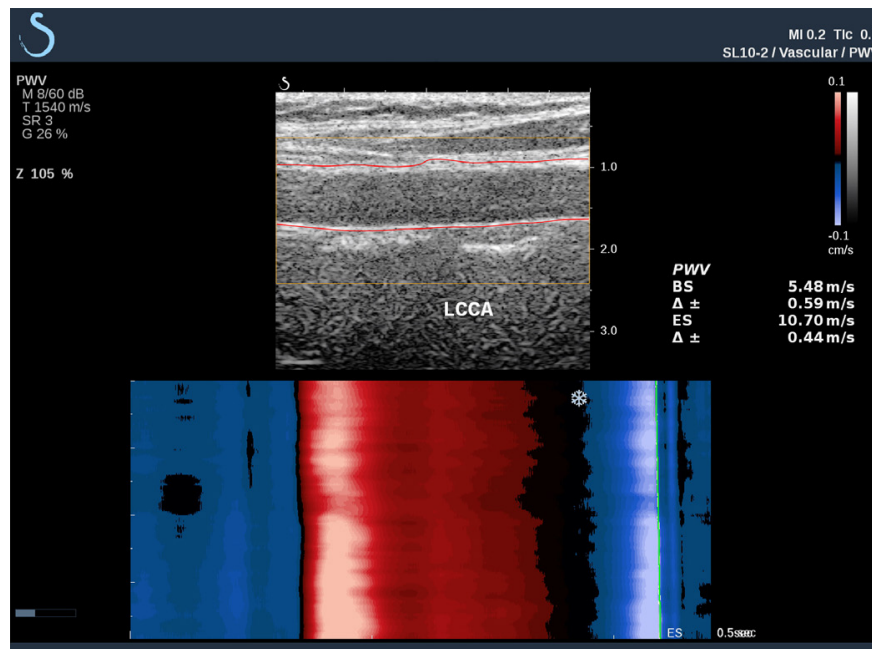

B

Fig. 2. Protocols for the valid assessment of cIMT using ACAMS and carotid stiffness using ufPWV in a 58-year-old woman.

A. Utilizing ACAMS, a white ROI box was drawn at the posterior wall of the CCA; the intimal and medial lines of the CCA were automatically recorded with two dotted lines, and the mean cIMT of the ROI was obtained with a fit of $83 \%$. B. Using ufPWV imaging, a yellow ROI box covered and a red line automatically tracked the anterior and posterior walls of the CCA. The PWV-BS and PWV-ES values were calculated in the ROI with $\triangle \pm$ of $\leq 1.0 \mathrm{~m} / \mathrm{s}$. The abscissa in the color graphs below the images represent time (ms), and the vertical axis represents the anterior CCA wall distance $(\mathrm{mm})$. Red shows movement towards the probe, and blue shows movement away from the probe. The PWV-BS value is $5.48 \mathrm{~m} / \mathrm{s}$, and the PWV-ES value is $10.70 \mathrm{~m} / \mathrm{s}$. CIMT, carotid intima-media thickness; ACAMS, Aixplorer cIMT automatic measurement system; ufPWV, ultrafast pulse wave velocity; ROI, region of interest; CCA, common carotid artery; LCCA, left common carotid artery; PWV$\mathrm{BS}$, pulse wave velocity-beginning of systole; PWV-ES, pulse wave velocity-end of systole.

conducted to calculate crude and adjusted odds ratios (ORs) with $95 \%$ confidence intervals, respectively. The candidate adjusted variables in the multivariable models were age, sex, smoking, SBP, and TC, which were major CVRFs in the SCORE assessment system. A P-value of $<0.05$ was considered to indicate statistical significance.

\section{Results}

\section{Baseline Characteristics of the Study Cohort}

Our study consisted of 683 individuals (mean age, 53.4 \pm 7.1 years), of whom $50.4 \%$ were women ( $n=344$; mean age, $52.5 \pm 7.0$ years), and $49.6 \%$ were men ( $n=339 ; 54.3 \pm 7.2$ years). Baseline demographic, clinical, and laboratory findings are summarized in Table 1. All measurements differed significantly according to sex subgroups (all $P<0.05$ ). Men had a notably higher body mass index, current smoking proportion, SBP, DBP, hemoglobin, fasting blood glucose, uric acid, LDL, TG, and TC levels, but a lower eGFR and HDL than women (all $P<0.05$ ).

\section{Associations between Age, Sex, cIMT, and Carotid Stiffening} Table 2 shows that men had a remarkably higher cIMT $(P<0.001)$, PWV-BS ( $P=0.005)$, and PWV-ES $(P=0.028)$ than women. To assess the association between cIMT, carotid stiffening and age, respectively, the middle-aged participants were further subdivided into four age subgroups as follows: $40-49$ years $(n=200$ [29.3\%]; men, $n=85$; women, $n=115$; as a control group), $50-54$ years $(n=162[23.7 \%]$; men, $n=70$; women, $n=92), 55-59$ years $(n=147$ [21.5\%]; men, $n=82$; women, $n=65), 60-65$ years $(n=174$ [25.5\%]; men, $n=102$; women, $n=72$ ). Compared with the 40 - to 49 year subgroup, men and women in the 50- to 65-year subgroups demonstrated markedly higher cIMT values (all $P<0.001$ ), except for 50- to 54-year-old men ( $P=0.275)$ (Fig. 3A). Participants in the 55- to 59-year subgroup had significantly higher PWV-BS values than control participants (all $P<0.05)$ (Fig. 3B). However, PWV-ES gradually increased with age in both men and women (all $P<0.001$ ) (Fig. 3C). Furthermore, $c I M T(r=0.408, P<0.001)$, PWV-BS ( $r=0.152$, $P<0.001)$, and PWV-ES $(r=0.429, P<0.001)$ were all positively correlated with age in middle-aged participants (Table 3).

\section{Relationship of SCORE with Age, Sex, cIMT, and Carotid Stiffening}

The proportions of SCORE-estimated risk differed significantly between men and women in this cohort $(\mathrm{P}<0.001)$ (Table 2). Men and women both had the highest proportion in the $1 \%-4 \%$ risk subgroup ( $67.3 \%$ for men; $55.5 \%$ for women). The second-highest 
Table 1. Baseline and laboratory characteristics of the study cohort

\begin{tabular}{|c|c|c|c|c|}
\hline & $\begin{array}{c}\text { Male } \\
(n=339)\end{array}$ & $\begin{array}{l}\text { Female } \\
(n=344)\end{array}$ & $\begin{array}{c}\text { Total } \\
(n=683)\end{array}$ & P-value \\
\hline \multicolumn{5}{|l|}{ Baseline characteristic } \\
\hline Age (year) & $54.3 \pm 7.2$ & $52.5 \pm 7.0$ & $53.4 \pm 7.1$ & 0.001 \\
\hline $\mathrm{BMI}\left(\mathrm{kg} / \mathrm{m}^{2}\right)$ & $23.8 \pm 3.1$ & $23.2 \pm 2.9$ & $23.5 \pm 3.0$ & 0.006 \\
\hline Current smoking & $102(30.1)$ & $1(0.3)$ & $103(15.1)$ & $<0.001$ \\
\hline $\mathrm{SBP}(\mathrm{mmHg})$ & $131.6 \pm 19.0$ & $127.2 \pm 19.2$ & $129.4 \pm 19.2$ & 0.001 \\
\hline $\mathrm{DBP}(\mathrm{mmHg})$ & $80.2 \pm 11.5$ & $76.4 \pm 11.7$ & $78.3 \pm 11.8$ & $<0.001$ \\
\hline \multicolumn{5}{|l|}{ Laboratory finding } \\
\hline Hemoglobin (g/L) & $143.7 \pm 22.8$ & $132.5 \pm 25.3$ & $138.0 \pm 24.7$ & $<0.001$ \\
\hline $\begin{array}{l}\text { Fasting blood } \\
\text { glucose (mmol/L) }\end{array}$ & $5.4 \pm 1.3$ & $5.2 \pm 0.9$ & $5.3 \pm 1.1$ & 0.040 \\
\hline Uric acid (mmol/L) & $350.5 \pm 94.5$ & $260.7 \pm 86.2$ & $306.2 \pm 101.0$ & $<0.001$ \\
\hline $\begin{array}{l}\text { eGFR } \\
\left(\mathrm{mL} / \mathrm{min} / 1.73 \mathrm{~m}^{2}\right)\end{array}$ & $88.1 \pm 28.4$ & $94.6 \pm 22.0$ & $91.4 \pm 25.5$ & $<0.001$ \\
\hline $\begin{array}{l}\text { LDL cholesterol } \\
(\mathrm{mmol} / \mathrm{L})\end{array}$ & $2.8 \pm 0.8$ & $2.6 \pm 0.8$ & $2.7 \pm 0.8$ & 0.003 \\
\hline $\begin{array}{l}\text { HDL cholesterol } \\
(\mathrm{mmol} / \mathrm{L})\end{array}$ & $1.3 \pm 0.3$ & $1.6 \pm 0.4$ & $1.4 \pm 0.4$ & $<0.001$ \\
\hline $\mathrm{TG}(\mathrm{mmol} / \mathrm{L})$ & $1.6 \pm 1.1$ & $1.3 \pm 0.9$ & $1.4 \pm 1.0$ & $<0.001$ \\
\hline TC (mmol/L) & $4.9 \pm 1.0$ & $4.4 \pm 1.0$ & $4.7 \pm 1.1$ & $<0.001$ \\
\hline
\end{tabular}

Values are presented as mean \pm SD or number (\%).

BMI, body mass index; SBP, systolic blood pressure; DBP, diastolic blood pressure; eGFR, estimated glomerular filtration rate; LDL, low-density lipoprotein; HDL, highdensity lipoprotein; TG, triglyceride; TC, total cholesterol.

Table 2. cIMT, ufPWV, and SCORE of the study cohort

\begin{tabular}{lcccc}
\hline & $\begin{array}{c}\text { Male } \\
(\mathrm{n}=339)\end{array}$ & $\begin{array}{c}\text { Female } \\
(\mathrm{n}=344)\end{array}$ & $\begin{array}{c}\text { Total } \\
(\mathrm{n}=683)\end{array}$ & P-value \\
\hline $\begin{array}{l}\text { Carotid ultrasound } \\
\text { finding }\end{array}$ & & & & \\
$\quad$ CIMT (cm) & $0.057 \pm 0.011$ & $0.054 \pm 0.009$ & $0.055 \pm 0.010$ & $<0.001$ \\
$\quad$ Range & $0.043-0.097$ & $0.043-0.100$ & $0.043-0.100$ & \\
PWV-BS (m/s) & $6.37 \pm 1.22$ & $6.12 \pm 1.08$ & $6.24 \pm 1.16$ & 0.005 \\
$\quad$ Range & $3.23-10.39$ & $3.57-10.73$ & $3.23-10.73$ & \\
PWV-ES (m/s) & $8.67 \pm 1.83$ & $8.43 \pm 1.82$ & $8.50 \pm 1.83$ & 0.028 \\
$\quad$ Range & $4.96-13.84$ & $4.06-12.78$ & $4.06-13.84$ & \\
SCORE (\%) & & & & $<0.001$ \\
$<1$ & $37(10.9)$ & $152(44.2)$ & $189(27.7)$ & \\
$1-4$ & $228(67.3)$ & $191(55.5)$ & $419(61.3)$ & \\
$5-9$ & $59(17.4)$ & $1(0.3)$ & $60(8.8)$ & \\
$>10$ & $15(4.4)$ & 0 & $15(2.2)$ & \\
\hline
\end{tabular}

Values are presented as mean \pm SD or number (\%) unless otherwise indicated. CIMT, carotid intima-media thickness; ufPWV, ultrafast pulse wave velocity; SCORE, Systematic COronary Risk Estimation; PWV-BS, pulse wave velocity at the beginning of systole; PWV-ES, pulse wave velocity at the end of systole. ratio was observed in the $5 \%-9 \%$ risk subgroup for men $(17.4 \%$ vs. $0.3 \%$ in women) and in the $<1 \%$ risk subgroup for women ( $44.2 \%$ vs. $10.9 \%$ in men). In addition, one female participant was classified as belonging to the $5 \%-9 \%$ risk subgroup, and none were in the $>10 \%$ risk subgroup (vs. $4.4 \%$ in men) (Table 2). The SCORE-estimated risk proportion gradually increased with age in women and men in this cohort (Fig. 4A, B). Furthermore, compared with the $<1 \%$ risk subgroup, participants with $\geq 1 \%$ risk had a greater cIMT (all $\mathrm{P}<0.05$ ), except for the $5 \%-9 \%$ risk subgroup in women $(P=0.193)$ (Fig. $5 A)$. Only in the $1 \%-4 \%$ risk subgroup, PWV-BS was higher than in the $<1 \%$ risk subgroup (all $\mathrm{P}<0.05$ ) (Fig. $5 B)$. However, PWV-ES gradually increased in all $\geq 1 \%$ risk subgroups for both sexes (all $P<0.001$, except for the $5 \%-9 \%$ risk subgroup, where $\mathrm{P}=0.012$ ) (Fig. $5 \mathrm{C}$ ). In total, some major CVRFs utilized in the SCORE assessment were correlated with PWV-BS for TC $(r=0.093$, $P=0.015)$, while cIMT $(r=0.306, P<0.001)$ and PWV-ES $(r=0.340$, $P<0.001)$ were correlated with SBP (Table 3). Furthermore, cIMT $(r=0.388, P<0.001)$, PWV-BS $(r=0.159, P<0.001)$, and PWV-ES $(r=0.405, P<0.001)$ were all positively correlated with SCOREestimated risk in middle-aged participants (Table 3).

\section{Predictive Value of Increased ufPWV for SCORE-Estimated Risk Progression}

Utilizing the low-risk subgroup as the reference control, univariable analyses showed significant associations between carotid stiffening and SCORE-estimated risk with PWV-BS (OR, 1.51 and OR, 1.36 for moderate-risk and high-risk subgroups, respectively, all $P<0.001$, and $P=0.824$ in the very-high-risk subgroup) and PWV-ES (OR, 1.61; $O R, 2.05$; and $O R, 1.95$ in the moderate-risk, high-risk, and very-high-risk subgroups, respectively, all $\mathrm{P}<0.001$ ) (Table 4). In multivariable models adjusted for sex, SBP, TC, and smoking, higher PWV-BS values were associated with SCORE-estimated risk only in the moderate-risk subgroup $(O R, 1.37 ; P=0.002)$, but not in the high-risk or very-high-risk subgroups $(\mathrm{P}=0.747$ and $\mathrm{P}=0.994$, respectively) (Table 4 ). With the same adjusted conditions, higher PWV-ES values were associated with SCORE-estimated risk in the moderate-risk $(O R, 1.63 ; P<0.001)$ and high-risk $(O R, 2.12$; $P=0.024)$ subgroups, but not in the very-high-risk subgroup $(P=0.992)$ (Table 4). However, the predictive values of increased PWV-BS and PWV-ES for SCORE-estimated risk progression were nonindependent of age in all $\geq 1 \%$ risk subgroups (all $P>0.05$ ) (Table 4).

\section{Discussion}

It is widely accepted that age, sex, high blood pressure, smoking, and dyslipidemia are major CVRFs, which cluster and interact multiplicatively to foster the development of cardiovascular disease 

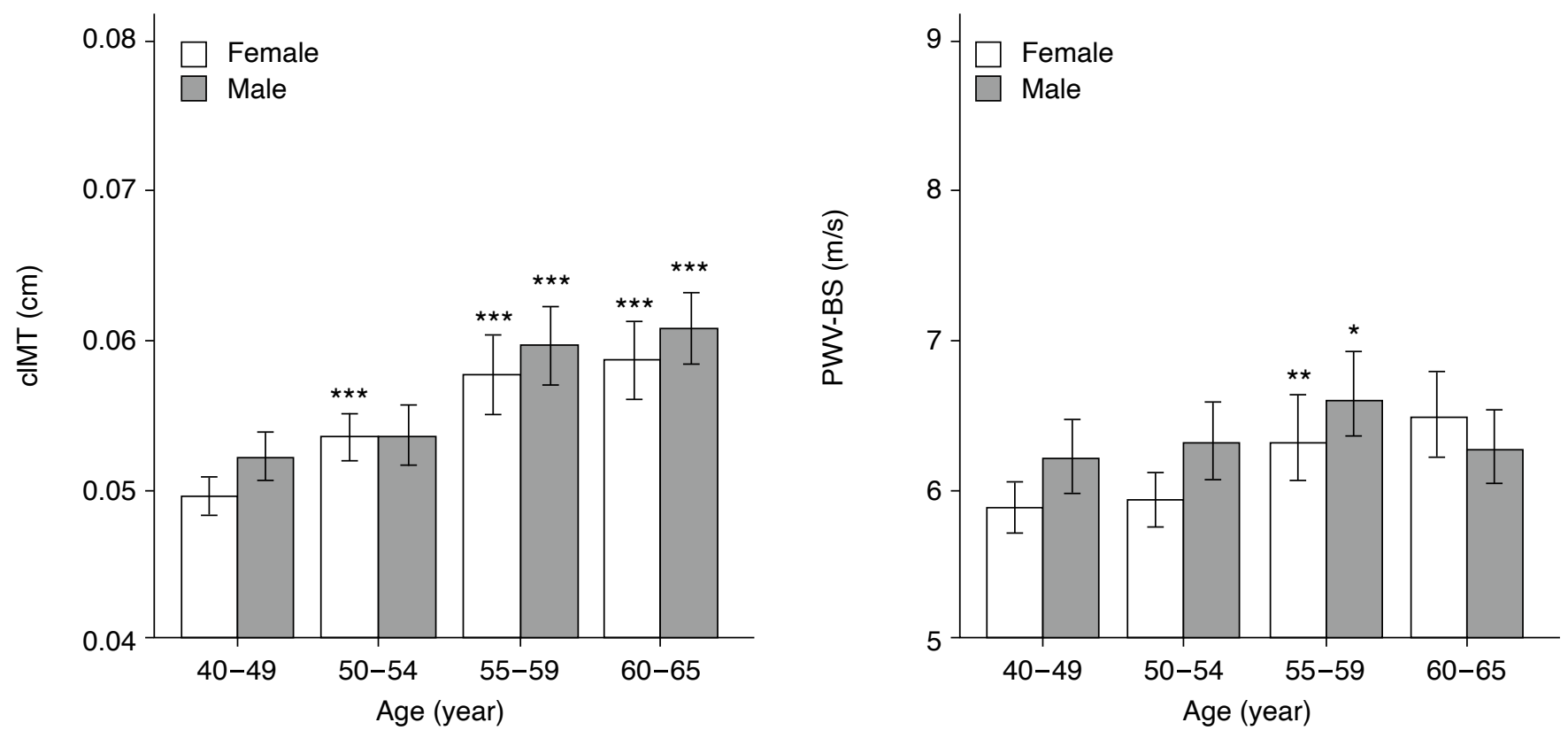

A

B

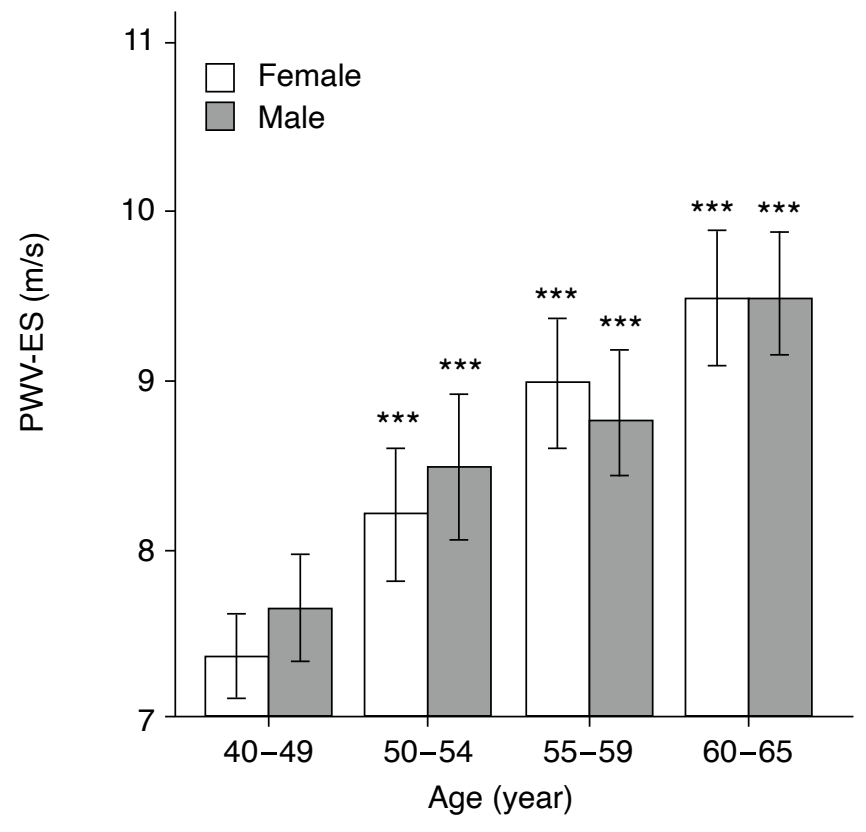

Fig. 3. Histograms showing comparisons of measurements of CIMT (A), PWV-BS (B), and PWV-ES (C) between age subgroups, respectively.

All comparisons were made with the 40- to 49-year subgroup in both men and women. cIMT, carotid intima-media thickness; PWV$B S$, pulse wave velocity-beginning of systole; PWV-ES, pulse wave velocity-end of systole. ${ }^{*} P<0.05,{ }^{*} P<0.01,{ }^{* *} P<0.001$.

C

Table 3. Correlations between cIMT, ufPWV, major cardiovascular risk factors, and SCORE

\begin{tabular}{|c|c|c|c|c|c|c|}
\hline & \multicolumn{2}{|c|}{$\mathrm{cIMT}(\mathrm{cm})$} & \multicolumn{2}{|c|}{ PWV-BS (m/s) } & \multicolumn{2}{|c|}{ PWV-ES (m/s) } \\
\hline & $r$ & P-value & $r$ & P-value & $r$ & P-value \\
\hline Age (year) & 0.408 & $<0.001$ & 0.152 & $<0.001$ & 0.429 & $<0.001$ \\
\hline TC (mmol/L) & 0.021 & 0.578 & 0.093 & 0.015 & 0.037 & 0.338 \\
\hline SCORE (\%) & 0.388 & $<0.001$ & 0.159 & $<0.001$ & 0.405 & $<0.001$ \\
\hline
\end{tabular}

CIMT, carotid intima-media thickness; ufPWV, ultrafast pulse wave velocity; SCORE, Systematic COronary Risk Estimation; PWV-BS, pulse wave velocity at the beginning of systole; PWV-ES, pulse wave velocity at the end of systole; SBP, systolic blood pressure; TC, total cholesterol. 
and to promote atherogenesis risk [24]. The main findings of this study are that in apparently healthy participants without established cardiovascular disease or diabetes mellitus, ufPWV was positively associated with SCORE-estimated risk in middle-aged participants. In addition, the predictive role of PWV-ES was independent of some major CVRFs, except for age, highlighting an extraordinarily close link between arterial stiffening and aging. Consistent with this, previous studies have shown that elevated carotid-femoral PWV and estimated PWV were associated with subsequent mortality, cardiovascular morbidity, and SCORE, albeit not independently of some major CVRFs $[25,26]$. However, they do not support changing SCORE by including arterial stiffening in the risk equation, since estimated PWV does not improve the risk function based on conventional CVRFs, which are covered by SCORE [26]. Moreover, the findings of the present study show that ufPWV can differentiate low-risk individuals from individuals with SCORE categories indicating moderate risk, high risk, or very high risk. Of note, a 1.0 $\mathrm{m} / \mathrm{s}$ increase in PWV-ES indicated a low risk of significant coronary or carotid artery disease translating to a higher stage, with positive odds showing a near doubling (OR, 1.61 to 2.05). This suggests
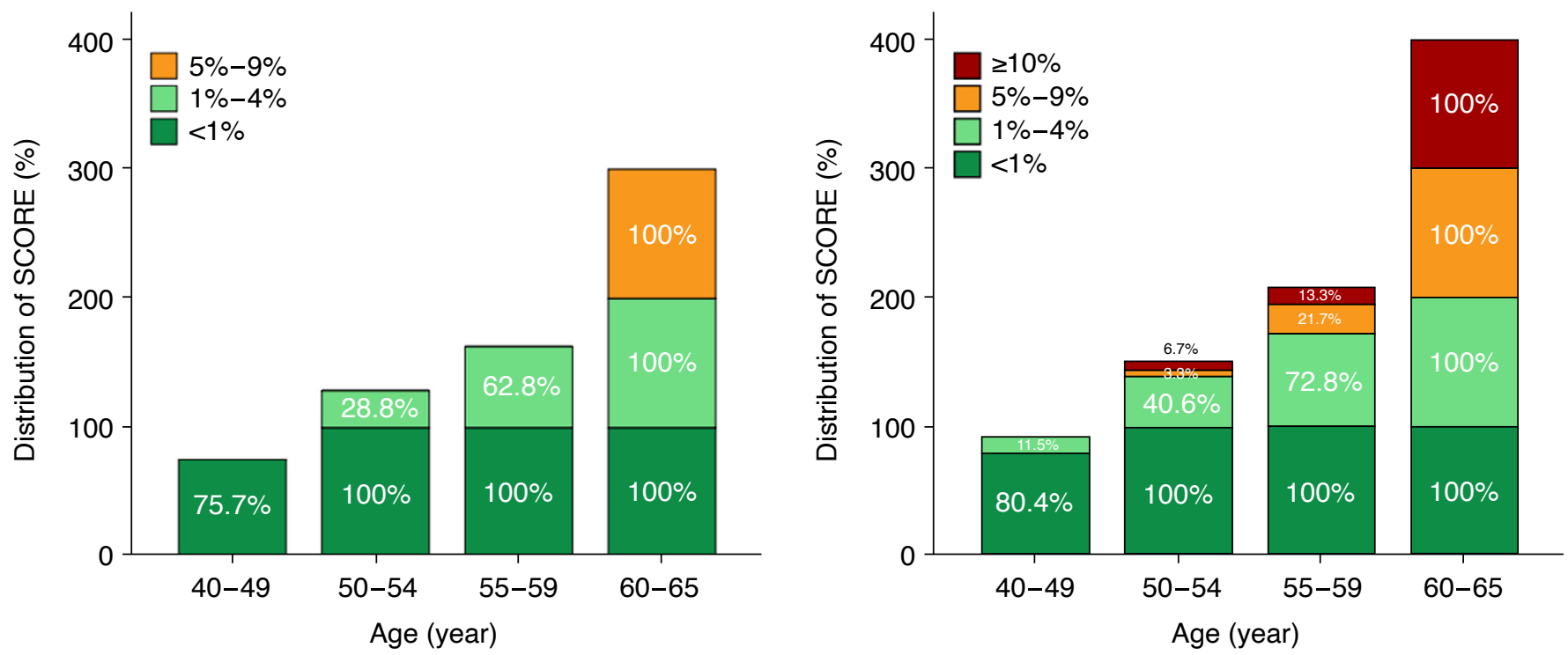

A

B

Fig. 4. Percent stacked area charts of SCORE risk categories of women $(n=344)(A)$ and men $(n=339)(B)$ in the age subgroups. SCORE, Systematic COronary Risk Evaluation.

Table 4. Odds ratios of PWV-BS and PWV-ES for SCORE classification

\begin{tabular}{|c|c|c|c|c|c|c|}
\hline & \multicolumn{2}{|c|}{ Moderate risk $(1 \%-4 \%, \mathrm{n}=419)$} & \multicolumn{2}{|c|}{ High risk $(5 \%-9 \%, n=60)$} & \multicolumn{2}{|c|}{ Very high risk $(\geq 10 \%, \mathrm{n}=15)$} \\
\hline & OR $(95 \% \mathrm{Cl})$ & P-value & OR $(95 \% \mathrm{Cl})$ & P-value & OR $(95 \% \mathrm{Cl})$ & P-value \\
\hline \multicolumn{7}{|l|}{ Model 1} \\
\hline PWV-BS, $\times 1 \mathrm{~m} / \mathrm{s}$ & $1.51(1.27-1.78)$ & $<0.001$ & $1.36(1.03-1.78)$ & 0.029 & $1.06(0.63-1.78)$ & 0.824 \\
\hline PWV-ES, $\times 1 \mathrm{~m} / \mathrm{s}$ & $1.61(1.43-1.81)$ & $<0.001$ & $2.05(1.66-2.52)$ & $<0.001$ & $1.95(1.41-2.69)$ & $<0.001$ \\
\hline \multicolumn{7}{|l|}{ Model 2} \\
\hline PWV-BS, $\times 1 \mathrm{~m} / \mathrm{s}$ & $1.37(1.13-1.66)$ & 0.002 & $0.88(0.40-1.92)$ & 0.747 & - & 0.994 \\
\hline PWV-ES, $\times 1 \mathrm{~m} / \mathrm{s}$ & $1.63(1.42-1.87)$ & $<0.001$ & $2.12(1.11-4.08)$ & 0.024 & - & 0.992 \\
\hline \multicolumn{7}{|l|}{ Model 3} \\
\hline PWV-BS, $\times 1 \mathrm{~m} / \mathrm{s}$ & $0.94(0.65-1.37)$ & 0.751 & - & $>0.99$ & - & $>0.99$ \\
\hline PWV-ES, $\times 1 \mathrm{~m} / \mathrm{s}$ & $1.08(0.87-1.34)$ & 0.477 & - & $>0.99$ & - & $>0.99$ \\
\hline
\end{tabular}

All ORs were calculated with the low-risk subgroup $(<1 \%, \mathrm{n}=189)$ as the reference. Model 1: crude OR; model 2: model 1+sex, systolic blood pressure, total cholesterol, and smoking; model 3: model 2+age.

PWV-BS, pulse wave velocity at the beginning of systole; PWV-ES, pulse wave velocity at the end of systole; SCORE, Systematic COronary Risk Estimation; OR, odds ratio; CI, confidence interval; -, non-significant. 
that ufPWV might be a useful add-on to SCORE in routine clinical practice. Of course, sufficient and substantial validation of the technique, prognostic information, and cohort dependency are first required.

Age is a vital marker of atherogenesis for duration of exposure to known and unknown CVRFs [27]. In this study, a stronger correlation was observed between carotid stiffening and age than between carotid stiffening and SCORE categories or other CVRFs. In

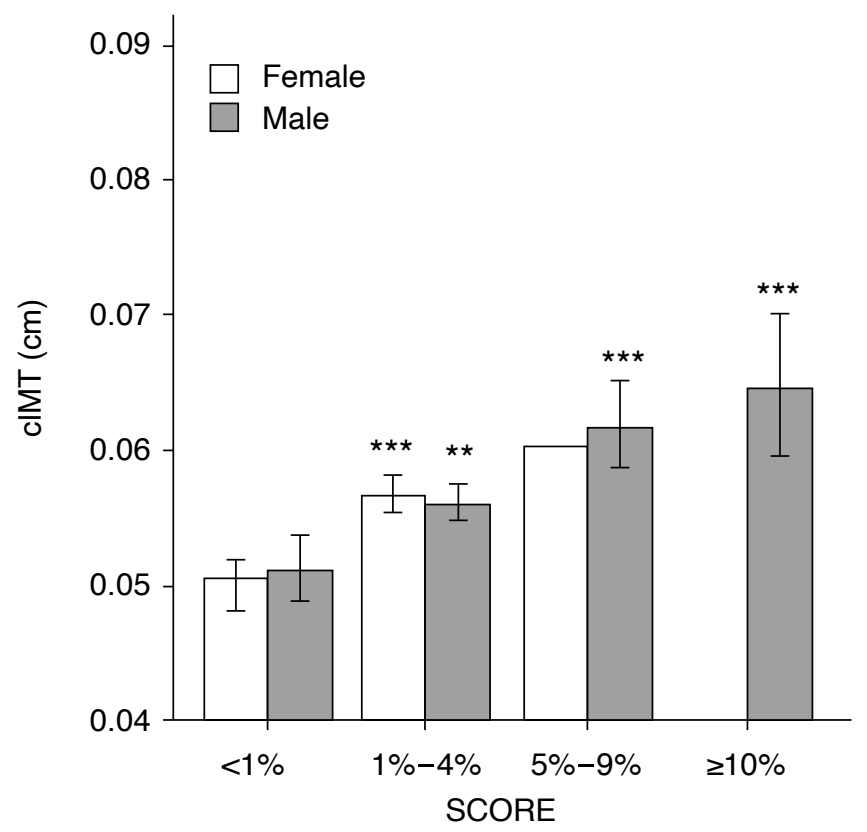

A

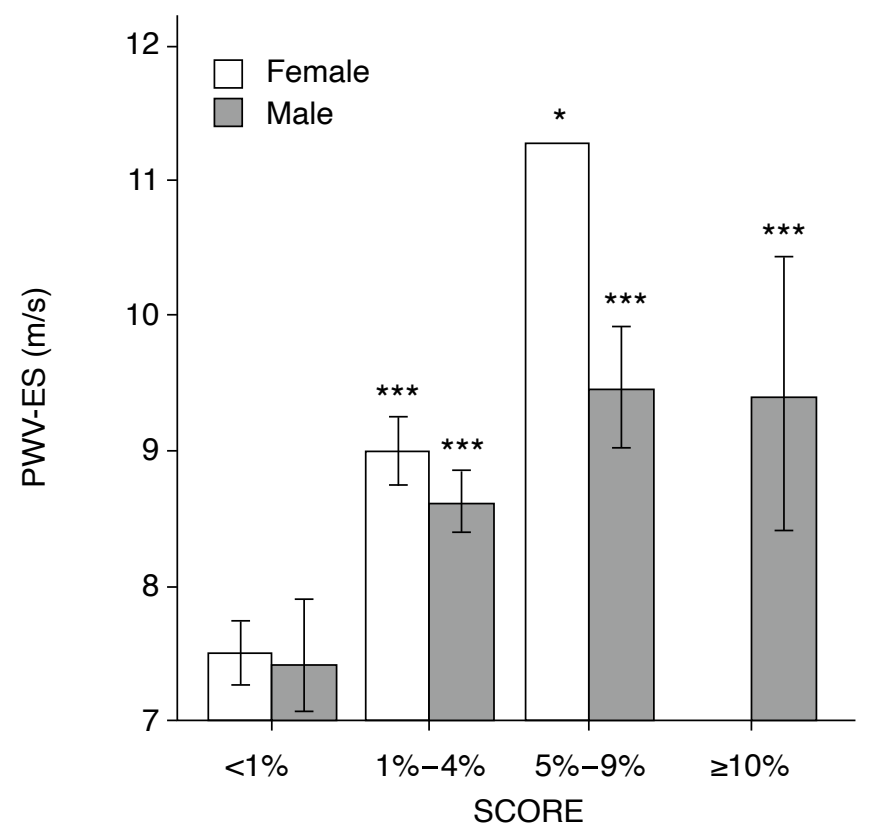

fact, this correlation was weak when considering previous studies utilizing ufPWV (0.304-0.476 for PWV-BS and age; 0.682-0.721 for PWV-ES and age) $[17,21,28]$. This is likely related to the middleaged population incorporated in this cohort, which had a limited age range of 40-65 years. However, recent evidence has reinforced the potential role of the SCORE risk chart to estimate the risk of atherosclerosis in its very early stages [6], which at present are proven to be covered by ufPWV assessments $[17,21]$, yielding that

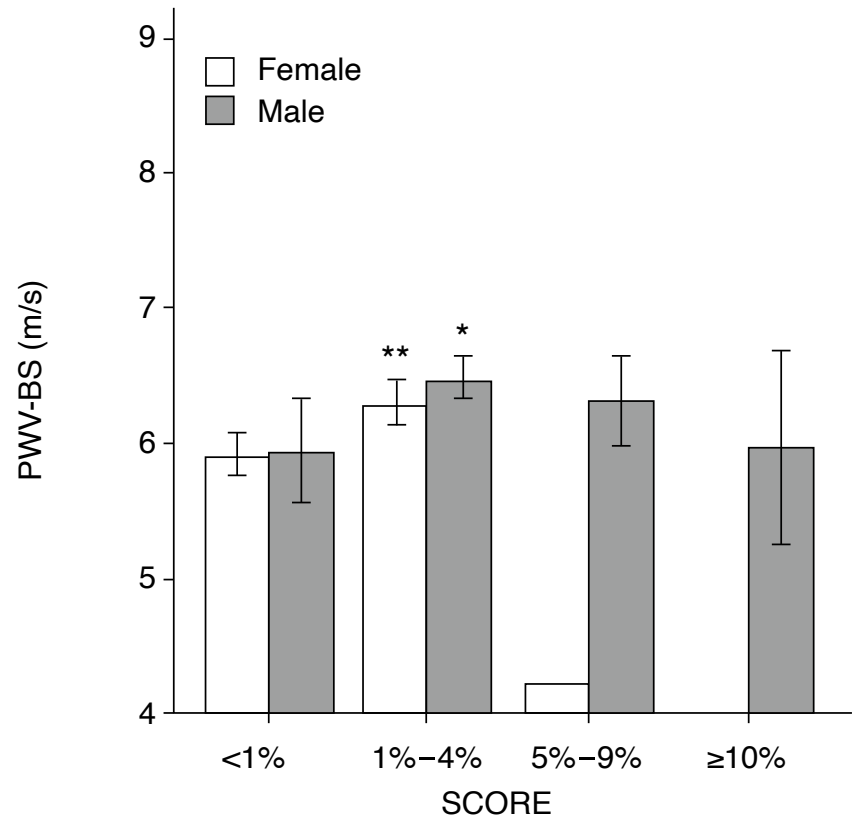

B

Fig. 5. Histograms showing comparisons of measurements of CIMT (A), PWV-BS (B), and PWV-ES (C) between SCORE subgroups.

All comparisons were made with the low-risk subgroup $(<1 \%)$ in both men and women. Of note, there was one woman in the $5 \%$ $9 \%$ risk subgroup, and no women in the $\geq 10 \%$ risk subgroup. cIMT, carotid intima-media thickness; PWV-BS, pulse wave velocitybeginning of systole; PWV-ES, pulse wave velocity-end of systole; SCORE, Systematic COronary Risk Evaluation. ${ }^{*} P<0.05$, ${ }^{*} P<0.01$, $* * * P<0.001$.

C 
the SCORE algorithm may be applied to younger individuals in the future. Furthermore, the better predictive value of PWV-ES rather than PWV-BS might be due to the substantial difference in wall recoil during a cardiac cycle, since early systolic expansions powered by left ventricular ejection may hinder the detection of minute differences in wall stiffness.

Sex differences also play a crucial role in atherogenesis due to the protective effect of endogenous estrogen in women [29]. The present study found that CIMT, carotid stiffening, SCORE categories, and major CVRFs all differed notably between sexes, whereas they all had the same trend in age-related atherosclerotic progression in both sexes. In this cohort, the majority of women were postmenopausal (214/344 [62.6\%]) and in the moderate SCORE risk group (191/344 [55.5\%]). Thus, differences in sex might not be interpretable in terms of the effects of estrogen alone. Modern epidemiological data [30] show that young women are relatively well-protected from cardiovascular disease, but women catch up to men at the age of $60-79$ years and ultimately surpass men by the age of 80 years, suggesting an interaction between sex and age. Of note, the distinct SCORE algorithms for men and women suggest underlying biological variation between sexes [4] and may highlight the need to include sex as an important component of individualized thresholds for prospective ufPWV assessment.

As a well-established marker of atherogenesis risk assessment, conventional cIMT is a composite measure of both intimal and medial layers in ultrasound screening. cIMT therefore represents a combination of atherosclerotic changes within the intimal layer and microstructural remodeling within the medial layer [31]. However, a lack of standardization in the definition and measurement of cIMT, its high variability, and its low intra-individual reproducibility have raised concerns. Large-scale meta-analyses [10,32] have failed to demonstrate any added value of cIMT in predicting cardiovascular disease and events. Additionally, detectable morphological alterations within atherosclerotic arterial walls usually lag behind biomechanical changes, such as augmented stiffness, especially in the subclinical phase with a normal cIMT and no plaques $[8,12,33]$. Consistent with this, the authors' prior studies have shown that individuals with non-elevated cIMT [17] or with no major CVRFs [21] still had carotid stiffening, as quantified by ufPWV. In the present analysis, PWV-ES was correlated more strongly than CIMT with SCORE categories, age, and major CVRFs. However, recent advances in ultra-high-frequency ultrasound modalities now permit the individual layers of the arterial wall to be studied in more detail $[34,35]$, which may change the present status of cIMT. However, this needs to be further validated.

This study has several limitations that should be noted. First, one potential limitation is the limited age range of patients in which
SCORE can be utilized. This limitation required us to assess arterial stiffening in a middle-aged population rather than in the general population. Research using ufPWV in younger participants has shown a reduction in carotid stiffness in individuals with optimally controlled CVRFs compared with ordinarily controlled CVRFs [21], suggesting that the superiority of ufPWV to CIMT might be underestimated in mid-life, restricting suitable candidates for ufPWV assessment. Second, although the possibility exists that different SCORE categories might not respond to risk factors in the same way, the predictive role of increased ufPWV with adjustment of major CVRFs was still validated in low-risk and high-risk groups in this study. Third, different ratios of SCORE categories might have led to cohort dependency, thereby affecting the validity of these results as extrapolated to the general population. Fourth, atherosclerosis is a life-long disease in which numerous factors play various roles. Some factors that may affect the cIMT and carotid stiffening were not considered in this study, such as lifestyle, alcohol consumption, medication, hyperthyroidism or hypothyroidism, other endocrinerelated diseases, genetic diseases, gynecological diseases, and anatomical variance in vascularity. Finally, ufPWV measurements were acquired from a single cohort using a single type of probe (SL10-2); thus, the cross-cohort and cross-probe generalizability of carotid stiffening remain to be tested.

In conclusion, the results of this study suggest that in vivo imaging of ufPWV is linked to SCORE categories, and that elevated PWV-ES may help to stratify differing levels of cardiovascular risk.

ORCID: Zhengqiu Zhu: https://orcid.org/0000-0002-2738-090X; Lingshan Chen: https://orcid.org/0000-0001-5159-7722; Wenjun Liu: https://orcid.org/0000-00024500-6559; Yiyun Wu: https://orcid.org/0000-0001-6402-929X; Chong Zou: https:// orcid.org/0000-0002-8727-6071; Xinyi Zhang: https://orcid.org/0000-0002-61930362; Shanshan He: https://orcid.org/0000-0002-0536-0844; Yinping Wang: https:// orcid.org/0000-0002-6273-5251; Bixiao Shen: https://orcid.org/0000-0001-63877991; Xuehui Ma: https://orcid.org/0000-0001-6449-0887; Hui Gao: https://orcid.org/00000003-4090-463X; Yun Luan: https://orcid.org/0000-0002-2953-0887; Hui Huang: https:// orcid.org/0000-0003-4319-8523

\section{※uthor affiliations}

Departments of ${ }^{1}$ Ultrasound and ${ }^{2}$ Radiology, Affiliated Hospital of Nanjing University of Chinese Medicine, Jiangsu Province Hospital of Chinese Medicine, Nanjing; ${ }^{3}$ School of Mathematics and Statistics, Nanjing University of Information Science and Technology, Nanjing; ${ }^{4}$ Department of Cardiology, Affiliated Hospital of Nanjing University of Chinese Medicine, Jiangsu Province Hospital of Chinese Medicine, Nanjing; ${ }^{5}$ Center of Good Clinical Practice, Affiliated Hospital of Nanjing University of Chinese Medicine, Jiangsu Province Hospital of Chinese Medicine, Nanjing, China 


\section{Author Contributions}

Conceptualization: Zhu Z, Huang H. Data acquisition: Zhu Z, Zhang $X$, He S, Wang Y, Shen B, Ma X, Gao H, Luan Y. Data analysis or interpretation: Zhu Z, Chen L, Liu W. Drafting of the manuscript: Zhu $Z$, Chen L, Huang H. Critical revision of the manuscript: Wu Y, Zou C, Huang $\mathrm{H}$. Approval of the final version of the manuscript: all authors.

\section{Conflict of Interest}

No potential conflict of interest relevant to this article was reported.

\section{Acknowledgments}

This work was supported by the National Natural Science Foundation of China (grant number: 81901756), the Key Research and Development Program of Jiangsu Province (grant number: BE2019725), and the Research Project of National Administration of Chinese Medicine (grant number: YB2015020).

\section{References}

1. Khot UN, Khot MB, Bajzer CT, Sapp SK, Ohman EM, Brener SJ, et al. Prevalence of conventional risk factors in patients with coronary heart disease. JAMA 2003;290:898-904.

2. Fernandez-Friera L, Fuster V, Lopez-Melgar B, Oliva B, GarciaRuiz JM, Mendiguren J, et al. Normal LDL-cholesterol levels are associated with subclinical atherosclerosis in the absence of risk factors. J Am Coll Cardiol 2017;70:2979-2991.

3. Erbel R, Delaney JA, Lehmann N, McClelland RL, Mohlenkamp S, Kronmal RA, et al. Signs of subclinical coronary atherosclerosis in relation to risk factor distribution in the Multi-Ethnic Study of Atherosclerosis (MESA) and the Heinz Nixdorf Recall Study (HNR). Eur Heart J 2008;29:2782-2791.

4. Piepoli MF, Hoes AW, Agewall S, Albus C, Brotons C, Catapano $A L$, et al. 2016 European Guidelines on cardiovascular disease prevention in clinical practice: The Sixth Joint Task Force of the European Society of Cardiology and other societies on cardiovascular disease prevention in clinical practice (constituted by representatives of 10 societies and by invited experts) developed with the special contribution of the European Association for Cardiovascular Prevention \& Rehabilitation (EACPR). Eur Heart J 2016;37:2315-2381.

5. Jorstad HT, Colkesen EB, Minneboo M, Peters RJ, Boekholdt SM, Tijssen JG, et al. The Systematic COronary Risk Evaluation (SCORE) in a large UK population: 10-year follow-up in the EPIC-Norfolk prospective population study. Eur J Prev Cardiol 2015;22:119-126.

6. Ostgren CJ, Soderberg S, Festin K, Angeras O, Bergstrom G, Blomberg $A$, et al. Systematic coronary risk evaluation estimated risk and prevalent subclinical atherosclerosis in coronary and carotid arteries: a population-based cohort analysis from the
Swedish Cardiopulmonary Bioimage Study. Eur J Prev Cardiol 2021;28:250-259.

7. Collins DR, Tompson AC, Onakpoya IJ, Roberts N, Ward AM, Heneghan CJ. Global cardiovascular risk assessment in the primary prevention of cardiovascular disease in adults: systematic review of systematic reviews. BMJ Open 2017;7:e013650.

8. Whitlock MC, Hundley WG. Noninvasive imaging of flow and vascular function in disease of the aorta. JACC Cardiovasc Imaging 2015;8:1094-1106.

9. Naqvi TZ, Lee MS. Carotid intima-media thickness and plaque in cardiovascular risk assessment. JACC Cardiovasc Imaging 2014;7:1025-1038.

10. Willeit P, Tschiderer L, Allara E, Reuber K, Seekircher L, Gao L, et al. Carotid intima-media thickness progression as surrogate marker for cardiovascular risk: meta-analysis of 119 clinical trials involving 100667 patients. Circulation 2020;142:621-642.

11. Libby P, Ridker PM, Hansson GK. Progress and challenges in translating the biology of atherosclerosis. Nature 2011;473:317325.

12. Reimann C, Brangsch J, Colletini F, Walter T, Hamm B, Botnar RM, et al. Molecular imaging of the extracellular matrix in the context of atherosclerosis. Adv Drug Deliv Rev 2017;113:49-60.

13. Authors/Task Force Members; Piepoli MF, Hoes AW, Agewall S, Albus C, Brotons C, et al. 2016 European Guidelines on cardiovascular disease prevention in clinical practice: The Sixth Joint Task Force of the European Society of Cardiology and Other Societies on Cardiovascular Disease Prevention in Clinical Practice (constituted by representatives of 10 societies and by invited experts) Developed with the special contribution of the European Association for Cardiovascular Prevention \& Rehabilitation (EACPR). Atherosclerosis 2016;252:207-274.

14. Vlachopoulos C, Aznaouridis K, Stefanadis C. Prediction of cardiovascular events and all-cause mortality with arterial stiffness: a systematic review and meta-analysis. J Am Coll Cardiol 2010;55:1318-1327.

15. Yin $L X$, Ma CY, Wang S, Wang YH, Meng PP, Pan XF, et al. Reference values of carotid Ultrafast pulse-wave velocity: a prospective, multicenter, population-based study. J Am Soc Echocardiogr 2021;34:629-641.

16. Marais L, Pernot M, Khettab H, Tanter M, Messas E, Zidi M, et al. Arterial stiffness assessment by shear wave elastography and Ultrafast pulse wave imaging: comparison with reference techniques in normotensives and hypertensives. Ultrasound Med Biol 2019;45:758-772.

17. Zhu ZQ, Chen LS, Wang H, Liu FM, Luan Y, Wu LL, et al. Carotid stiffness and atherosclerotic risk: non-invasive quantification with ultrafast ultrasound pulse wave velocity. Eur Radiol 2019;29:15071517.

18. Li $X$, Jiang J, Zhang $H$, Wang $H$, Han D, Zhou Q, et al. Measurement 
of carotid pulse wave velocity using ultrafast ultrasound imaging in hypertensive patients. J Med Ultrason (2001) 2017;44:183-190.

19. Pan FS, Xu M, Yu L, Luo J, Li MY, Liang JY, et al. Relationship between carotid intima-media thickness and carotid artery stiffness assessed by ultrafast ultrasound imaging in patients with type 2 diabetes. Eur J Radiol 2019;111:34-40.

20. Li Y, Zhang Y, Geng X, Zhao S, Sun YX, Wang YB. Increased carotid stiffness detected by ultrafast ultrasound imaging is associated with the Gensini score. Med Ultrason 2020;22:183-188.

21. Zhu ZQ, Chen LS, Jiang XZ, Wu YY, Zou C, Luan Y, et al. Absent atherosclerotic risk factors are associated with carotid stiffening quantified with ultrafast ultrasound imaging. Eur Radiol 2021;31:3195-3206.

22. Levey AS, Stevens LA, Schmid CH, Zhang YL, Castro AF 3rd, Feldman $\mathrm{HI}$, et al. A new equation to estimate glomerular filtration rate. Ann Intern Med 2009;150:604-612.

23. Stein JH, Korcarz CE, Hurst RT, Lonn E, Kendall CB, Mohler ER, et al. Use of carotid ultrasound to identify subclinical vascular disease and evaluate cardiovascular disease risk: a consensus statement from the American Society of Echocardiography Carotid IntimaMedia Thickness Task Force. Endorsed by the Society for Vascular Medicine. J Am Soc Echocardiogr 2008;21:93-111.

24. D'Agostino EB Sr, Vasan RS, Pencina MJ, Wolf PA, Cobain M, Massaro JM, et al. General cardiovascular risk profile for use in primary care: the Framingham Heart Study. Circulation 2008;117:743-753.

25. Greve SV, Blicher MK, Kruger R, Sehestedt T, Gram-Kampmann E, Rasmussen $\mathrm{S}$, et al. Estimated carotid-femoral pulse wave velocity has similar predictive value as measured carotid-femoral pulse wave velocity. J Hypertens 2016;34:1279-1289.

26. Vishram-Nielsen JK, Laurent S, Nilsson PM, Linneberg A, Sehested TS, Greve SV, et al. Does estimated pulse wave velocity add prognostic information?: MORGAM prospective cohort project. Hypertension 2020;75:1420-1428.
27. Tyrrell DJ, Goldstein DR. Ageing and atherosclerosis: vascular intrinsic and extrinsic factors and potential role of IL-6. Nat Rev Cardiol 2021;18:58-68.

28. Mirault T, Pernot M, Frank M, Couade M, Niarra R, Azizi M, et al. Carotid stiffness change over the cardiac cycle by ultrafast ultrasound imaging in healthy volunteers and vascular EhlersDanlos syndrome. J Hypertens 2015;33:1890-1896.

29. Man JJ, Beckman JA, Jaffe IZ. Sex as a Biological variable in atherosclerosis. Circ Res 2020;126:1297-1319.

30. Virani SS, Alonso A, Benjamin EJ, Bittencourt MS, Callaway CW, Carson AP, et al. Heart disease and stroke statistics-2020 update: a report from the American Heart Association. Circulation 2020;141:e139-e596.

31. Bots ML, Sutton-Tyrrell K. Lessons from the past and promises for the future for carotid intima-media thickness. J Am Coll Cardiol 2012;60:1599-1604.

32. Lorenz MW, Polak JF, Kavousi M, Mathiesen EB, Volzke H, Tuomainen TP, et al. Carotid intima-media thickness progression to predict cardiovascular events in the general population (the PROGIMT collaborative project): a meta-analysis of individual participant data. Lancet 2012;379:2053-2062.

33. Thondapu V, Bourantas CV, Foin N, Jang IK, Serruys PW, Barlis P. Biomechanical stress in coronary atherosclerosis: emerging insights from computational modelling. Eur Heart J 2017;38:81-92.

34. Chiesa ST, Charakida M, Georgiopoulos G, Dangardt F, Wade KH, Rapala $A$, et al. Determinants of Intima-media thickness in the young: the ALSPAC Study. JACC Cardiovasc Imaging 2021;14:468478.

35. Eklund C, Friberg P, Gan LM. High-resolution radial artery intimamedia thickness and cardiovascular risk factors in patients with suspected coronary artery disease: comparison with common carotid artery intima-media thickness. Atherosclerosis 2012;221:118-123. 